\title{
Evaluating Acid Resistance Effect of Fluoride-Releasing Dental Materials Using Quantitative Light-Induced Fluorescence-Digital in Vitro
}

\author{
Koji Watanabe*, Takashi Sasabe, Shigeru Watanabe \\ Division of Pediatric Dentistry, Department of Human Development and Fostering, Meikai University School of \\ Dentistry, Sakado, Japan \\ Email: "k-watanabe@dent.meikai.ac.jp
}

Received 14 March 2016; accepted 24 April 2016; published 27 April 2016

Copyright (C) 2016 by authors and Scientific Research Publishing Inc.

This work is licensed under the Creative Commons Attribution International License (CC BY). http://creativecommons.org/licenses/by/4.0/

(c) (7) Open Access

\section{Abstract}

As erosion has become one of the serious oral health problems, some fluoride-releasing dental materials have been developed to protect enamel from demineralization. The purpose of this study was to evaluate the antidemineralization effect of PRG Barrier Coat ${ }^{\circledR}$ and FujiVII ${ }^{\circledR}$ (fluoridereleasing dental materials). Four square windows measuring $1 \mathbf{~ m m}^{2}$ were prepared on the labial enamel surface of each bovine tooth using masking tape and nail varnish. The first and second windows were half covered by fluoride-releasing material and control material, respectively, whereas the third and fourth windows were left untreated. All windows were immersed into $0.1 \mathrm{M}$ lactic acid and $6 \mathrm{wt} \% \mathrm{CM}$-cellulose $\left(\mathrm{pH} \mathrm{4.5)}\right.$ at $38^{\circ} \mathrm{C}$ for 21 days. Subsequently, the first and second windows were covered with nail varnish after removal of the materials, and the third and fourth windows were immersed into ultrapure water at $38^{\circ} \mathrm{C}$ for 28 days after being half covered with fluoride-releasing and control materials, respectively. The following procedure was performed separately during evaluations of the fluoride-releasing materials, PRG Barrier Coat ${ }^{\circledR}$, and FujiVII ${ }^{\circledR}$, using eight and six bovine teeth, respectively. The first, second, third, and fourth windows were classified into DM-PRG; Demineralized-PRG or DM-FujiVII; Demineralized-FujiVII, DM-TCM; Demineralized-traditional cement or DM-TGIC; Demineralized-traditional glass ionomer cement, RM-PRG; Remineralized-PRG or RM-FujiVII; Remineralized-FujiVII, and RM-TCM; Remineralizedtraditional cement or RM-TGIC; Remineralized-traditional glass ionomer cement, respectively. After nail varnish was removed, $\Delta Q$ values (mean $\pm S D$ ) of the windows were measured using $Q L F-D$ and were compared between DM-PRG and DM-TCM, RM-PRG and RM-TCM, DM-FujiVII and DMTGIC, and RM-FujiVII and RM-TGIC groups. $\Delta Q$ values of RM-PRG $(-60 \pm 44)$ and RM-FujiVII $(-5.0$ $\pm 10)$ were significantly higher than that of RM-TCM $(-315 \pm 193)$ and RM-TGIC $(-56 \pm 43)$, re-

\footnotetext{
${ }^{*}$ Corresponding author.
}

How to cite this paper: Watanabe, K., Sasabe, T. and Watanabe, S. (2016) Evaluating Acid Resistance Effect of Fluoride-Releasing Dental Materials Using Quantitative Light-Induced Fluorescence-Digital in Vitro. Open Journal of Stomatology, 6, 127-134. http://dx.doi.org/10.4236/ojst.2016.64016 
spectively. The fluoride releasing materials provided remineralization effects to bovine enamel.

Keywords

\author{
Fluoride-Releasing Materials, Bovine Enamel, Erosion, Remineralization, Quantitative \\ Light-Induced Fluorescence-Digital
}

\title{
1. Introduction
}

As erosion has become one of the serious oral health problems, some fluoride-releasing dental materials have been developed to protect enamel from demineralization. Erosion is initiated as demineralization because of nonbacterial acid exposure such as that occurring during bulimia, gastric acid reflex, and frequent intake of acidic food [1] [2]. The importance of detection and control of early demineralized lesions by dentists is constantly increasing since severe carious lesions are currently not as common in Japan as during the past. In gener$\mathrm{al}$, an early demineralized lesion is treated with fluoride application, and the advantage of fluoride-releasing materials has already been reported [3]-[5]. However, to date, there has been insufficient reporting on the inhibition of demineralization and promotion of remineralization by fluoride-releasing materials. Quantitative Lightinduced Fluorescence-Digital (QLF-D; Inspektor, Amsterdam, The Netherlands) is a device used to evaluate severity or the time-course change of the early demineralized lesion by calculating mineral density [6] [7]. PRG Barrier Coat ${ }^{\circledR}$ is a light-cure coating material that contains surface pretreated glass (S-PRG) [8]. The S-PRG releases and recharges sodium, strontium, borate, aluminum, silicate, and fluoride ions depending on the oral surroundings, resulting in protective effects on early demineralized enamel. Glass ionomer cement is one of the materials that is applied to protect pulp and to fill cavities in dental treatment. It contains fluoroaluminosilicate glass that releases fluoride as one of the ingredients. FujiVII ${ }^{\circledR}$ is newly developed glass ionomer cement that possesses an improved fluoride-releasing property [3]. In this study, the antidemineralization effect of PRG Barrier Coat ${ }^{\circledR}$ and FujiVII ${ }^{\circledR}$ on bovine enamel was evaluated in vitro using QLF-D. The current study was approved by the ethical committee on experiments in Meikai University (approval number: A1305).

\section{Materials and Methods}

\subsection{Preparation of the Tooth Samples}

Eight and six bovine teeth for the evaluation of PRG Barrier Coat ${ }^{\circledR}$ and FujiVII ${ }^{\circledR}$, respectively, were polished by 400-, 600-, and 800-grit silicon carbide abrasive paper to remove extraneous matter from the enamel surface. Four square windows measuring $1 \mathrm{~mm}^{2}$ were prepared on the labial enamel surface of each tooth using masking tape and nail varnish (SUNPALKO Co., Ltd., Kyoto, Japan). The teeth were completely coated with nail varnish except for the window areas. The evaluation of PRG Barrier Coat ${ }^{\circledR}$ was carried out initially, following by the evaluation of FujiVII ${ }^{\circledR}$. Eight and six bovine teeth were available for the evaluation of PRG Barrier Coat ${ }^{\circledR}$ and FujiVII ${ }^{\circledR}$, respectively. As a result, the number of the samples used differed between the two evaluations of fluoride-releasing dental materials.

\subsection{Application of PRG Barrier Coat ${ }^{\circledR}$, FujiVII ${ }^{\circledR}$, and Control Materials before Demineralization}

For the eight bovine teeth, in the initial evaluation of PRG Barrier Coat ${ }^{\circledR}$, half of the window area was covered with PRG Barrier Coat ${ }^{\circledR}$ (a Demineralized-PRG;DM-PRG group) in one of the four windows, another window was covered with traditional tooth coating material in the same way (a Demineralized-traditional coating material; DM-TCM group), whereas the two remaining windows were left untreated (Figure 1(a)). The six bovine teeth for evaluation of FujiVII ${ }^{\circledR}$ were prepared in a similar way; half of the window area was covered with Fuji$\mathrm{VII}^{\circledR}$ (a Demineralized-FujiVII; DM-FujiVII group) in one of the four windows, another window was covered with traditional glass ionomer cement in the same way (a Demineralized-traditional glass ionomer cement; DM-TGIC group), whereas the remaining two windows were left untreated (Figure 1(b)). 


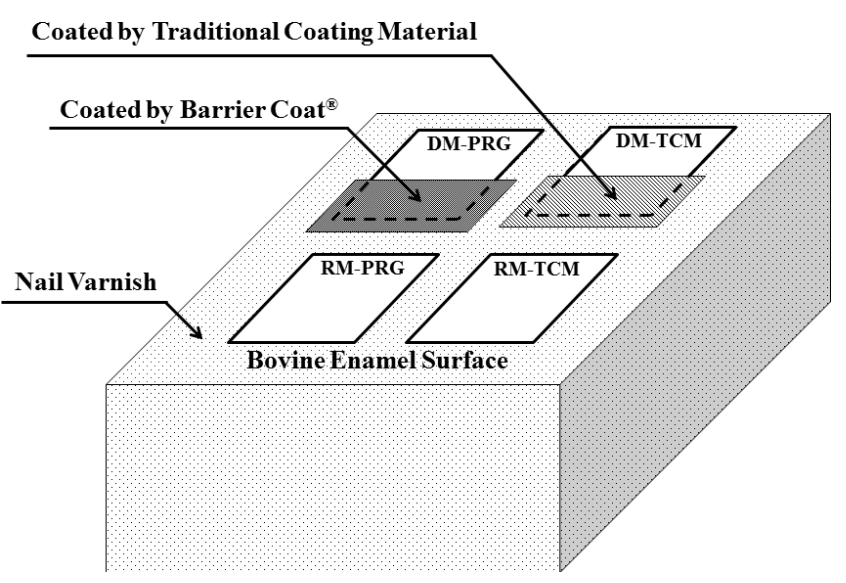

(a)

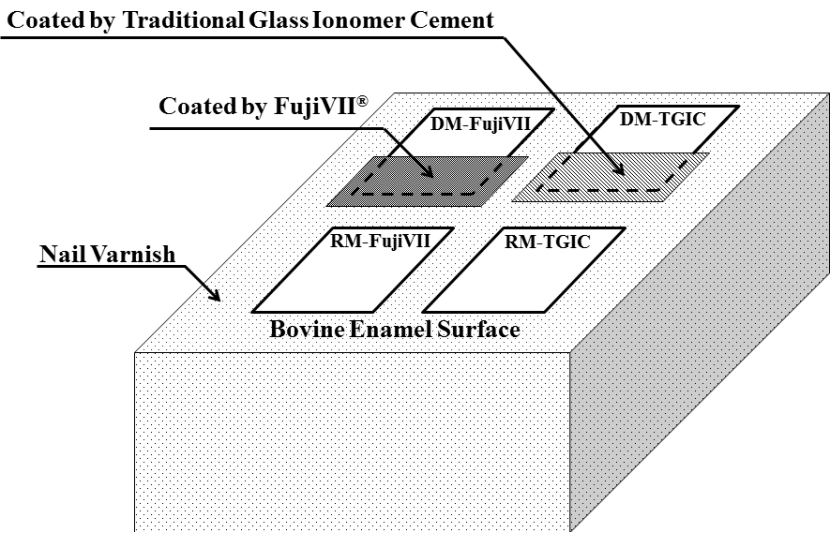

(b)

Figure 1. (a) Windows used for the evaluation of PRG Barrier Coat ${ }^{\circledR}$ during the step of demineralization. One of the four windows was covered with PRG Barrier Coat ${ }^{\circledR}$, another with traditional coating material, and the remaining two without any dental material. (b) Windows used for the evaluation of FijiVII ${ }^{\circledR}$ during the step of demineralization. One of the four windows was covered with FijiVII ${ }^{\circledR}$, another with traditional glass ionomer cement, and the remaining two without any dental material.

\subsection{Incubation of the Naked Window Areas in a Lactic Acid}

Tooth samples for evaluation of both PRG Barrier Coat ${ }^{\circledR}$ and FujiVII ${ }^{\circledR}$ were incubated in the same way. Every tooth sample was separately incubated in lactic acid solution that contained $0.1 \mathrm{M}$ of lactic acid and $6 \mathrm{wt} \%$ of CM-cellulose ( $\mathrm{pH}$ 4.5; Wako Pure Chemical Industries, Ltd., Osaka, Japan) at $38^{\circ} \mathrm{C}$ for 21 days. On the $21^{\text {st }}$ day of incubation, the tooth samples were removed from the lactic acid solution and washed with ultrapure water. After the PRG Barrier Coat ${ }^{\circledR}$ and traditional tooth coating material, or FujiVII ${ }^{\circledR}$ and traditional glass ionomer cement were removed from each tooth sample, the windows that had been incubated with the aforementioned dental materials were completely covered with nail varnish.

\subsection{Application of PRG Barrier Coat ${ }^{\circledR}$, FujiVII ${ }^{\circledR}$, and Control Materials before Remineralization}

In the eight teeth used in the evaluation of PRG Barrier Coat ${ }^{\circledR}$, one of the two windows that were immersed into the lactic acid solution without any dental material was half covered by PRG Barrier Coat ${ }^{\circledR}$ (a RemineralizedPRG; RM-PRG group), and the other window was half covered with traditional coating material (a Remineralized-traditional coating material; RM-TCM group), respectively (Figure 2(a)). In the six teeth used in the evaluation of FujiVII ${ }^{\circledR}$, one of the two windows that were immersed into the lactic acid solution without any dental material was half covered by FujiVII ${ }^{\circledR}$ (a Remineralized-FujiVII; RM-FujiVII group), and the other window was half covered with traditional glass ionomer cement (a Remineralized-traditional glass ionomer cement; 


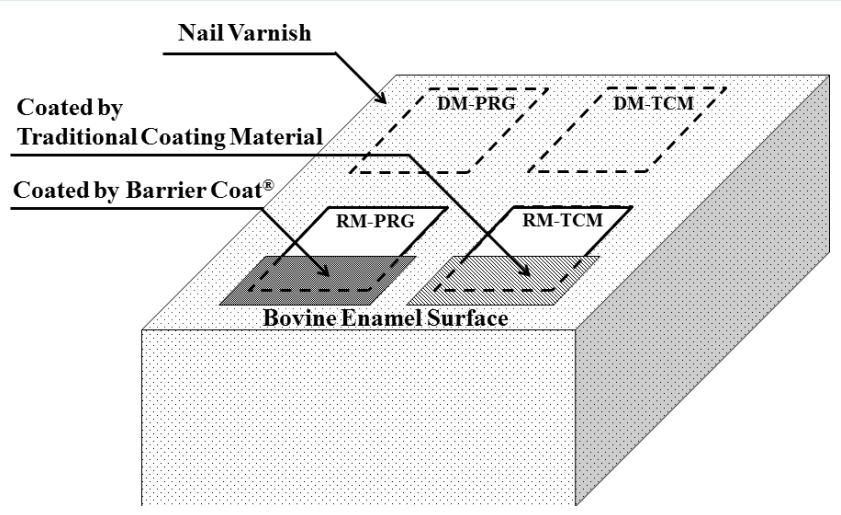

(a)

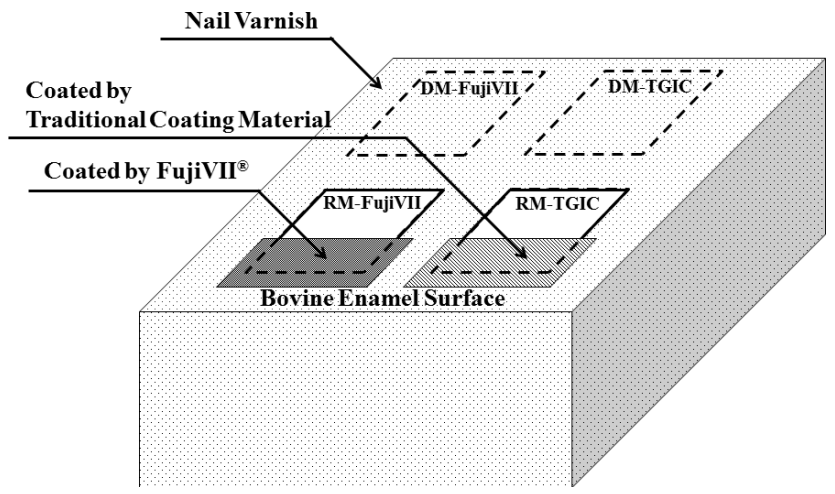

(b)

Figure 2. (a) Windows used for the evaluation of PRG Barrier Coat ${ }^{\circledR}$ during the step of remineralization. The two windows that were demineralized with dental material were completely covered with nail varnish. The remaining two windows that were demineralized without any dental material were half covered with PRG Barrier Coat ${ }^{\circledR}$ and traditional coating material, respectively. (b) Windows used for the evaluation of FijiVII ${ }^{\circledR}$ during the step of remineralization. The two windows that were demineralized with dental material were completely covered with nail varnish. The remaining two windows that were demineralized without any dental material were half covered with FijiVII ${ }^{\circledR}$ and traditional glass ionomer cement, respectively.

RM-TGIC group), respectively. All the tooth samples for the both evaluations were incubated in ultrapure water at $38^{\circ} \mathrm{C}$ for 10 days (Figure 2(b)). After the incubation, the tooth samples were washed with ultrapure water, following which all the materials and nail varnish on the tooth surface were removed (Figure 3 ).

\subsection{Measurement of Mineral Density}

The value of $\Delta \mathrm{Q}$ on the border of the dental material in each window area (Figure 4) was measured by QLF-D. The images were captured from the labial aspect of the sample teeth in a darkroom, using proprietary software (C3 v1.19, Inspektor Research Systems BV, Amsterdam, The Netherlands) under the following settings: shutter speed of 1/60s, aperture value of 5.6, and ISO speed of 1600 [6]. The distance between the lens and the window was $10 \mathrm{~cm}$ and the shooting angle was maintained as $90^{\circ}$ to the window surface. The mean values were compared between the DM-PRG and DM-TCM groups, DM-FujiVII and DM-TGIC groups, RM-PRG and RMTCM groups, and RM-FujiVII and RM-TGIC groups. A student's t-test was used to compare the significance of the difference between the two groups and $\mathrm{p}<0.05$ was considered significant.

\section{Results}

Values were expressed as mean \pm standard deviation. Window areas were demineralized or de- and remineralized hence turned in white without any parenchyma loss. QLF made possible to evaluate mineral density in early demineralized lesion. $\Delta \mathrm{Q}$ values in the window areas were evaluated under the condition described in the Materials and Method section. As shown in Table 1 and Table 2, the $\Delta Q$ value in the RM-PRG group ( $-60 \pm 44)$ 


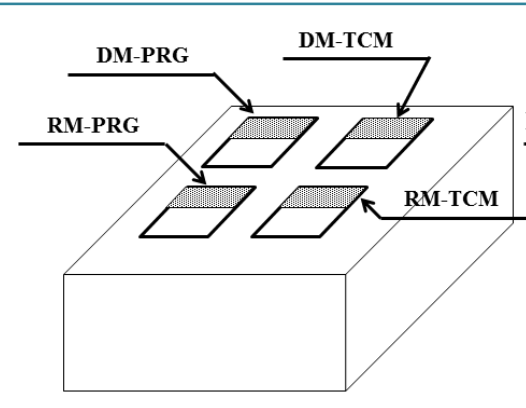

Windows of Evaluation for PRG Barrier Coat ${ }^{\mathbb{8}}$

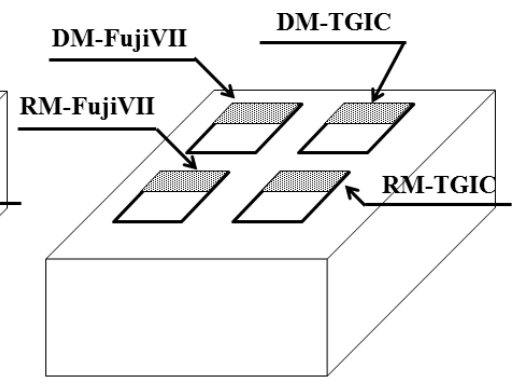

Windows of Evaluation for FujiVII ${ }^{\circledR}$

Figure 3. Four windows prepared for evaluations and measured area in the windows. The windows were classified into four groups in evaluation of each material.

\section{$\triangle \times \times \times x \times \times \square \quad$ : Measured Area}

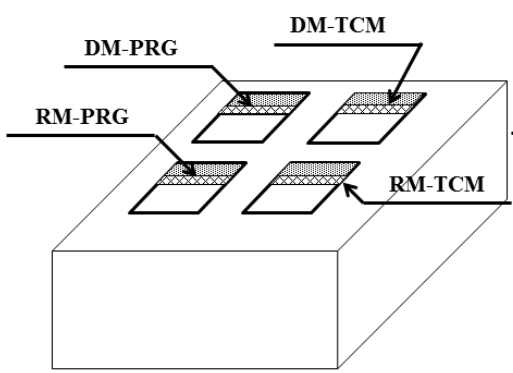

Windows of Evaluation for PRG Barrier Coat ${ }^{\text {B }}$

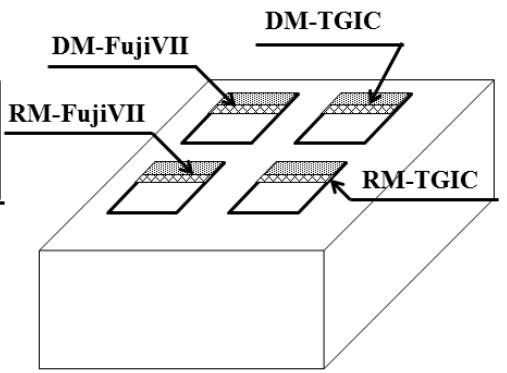

Windows of Evaluation for FujiVII ${ }^{\circledR}$

Figure 4. Measured area in the windows. Measurement of the $\Delta \mathrm{Q}$ values were carried out on the border of dental material in each window area.

Table 1. $\Delta \mathrm{Q}$ value of coating material groups.

\begin{tabular}{cc}
\hline Group & $\Delta$ Q Value (\%pixels) \\
\hline DM-TCM & $-787 \pm 683$ \\
DM-PRG & $-463 \pm 529$ \\
RM-TCM & $-315 \pm 193^{* *}$ \\
RM-PRG & $-60 \pm 44^{* *}$ \\
\hline${ }^{* *}<0.01$. &
\end{tabular}

Table 2. $\Delta \mathrm{Q}$ value in glass ionomer cement groups.

\begin{tabular}{cc}
\hline Group & $\Delta$ Q Value (\%pixels) \\
\hline DM-TGIC & $-25 \pm 36$ \\
DM-FujiVII & $-33 \pm 46$ \\
RM-TGIC & $-56 \pm 43^{*}$ \\
RM-FujiVII & $-5 \pm 10^{*}$ \\
${ }^{*} \mathrm{p}<0.05$. &
\end{tabular}

was significantly higher than that in the RM-TCM group $(-315 \pm 193 ; \mathrm{p}<0.01)$, and the $\Delta \mathrm{Q}$ value in the RM-FujiVII group $(-5 \pm 10)$ was also significantly higher than that in the RM-TGIC group $(-56 \pm 43$; $\mathrm{p}<0.05)$, respectively. 


\section{Discussion}

Lussi and Carvalho (2015) [9] mentioned in their study on fluorides and other protective agents against demineralization that the effectiveness of fluoride in caries prevention has been convincingly proven. In addition, both previous in vitro and clinical studies reported that fluoride improves remineralization [10] [11], supporting our results. Lippert et al. (2013) [12] reported in their in vitro study that fluoride reduced enamel demineralization in a mild lesion; however, application of fluoride-releasing materials did not reduce demineralization of enamel in the current study. It could be considered that $0.1 \mathrm{M}$ of lactic acid and $6 \mathrm{wt} \%$ of CM-cellulose (pH 4.5) as a demineralization solution resulted in a too severe condition for released fluoride to reduce demineralization of enamel. Moreover, ten Cate et al. (2006) [13] reported in their in vitro study on the correlation between artificially induced demineralized lesion depth and the concentration of topical fluoride application, that fluoride can drive demineralization further into enamel by resulting in less soluble surface tissue, by not neutralizing acids penetrating into the tissue, thereby supporting our results that mineral density in enamel was not affected by topical fluoride application during demineralization.

PRG Barrier Coat ${ }^{\circledR}$ is a tooth coating material that is often applied to cervical, contact, and caries prone areas to prevent tooth decay or erosion. It contains S-PRG ionomer as fillers, which releases and recharges $\mathrm{F}^{-}, \mathrm{Sr}^{2+}$, $\mathrm{Na}^{+}, \mathrm{SiO}_{3}^{2-}, \mathrm{Al}^{3+}$, and $\mathrm{BO}_{3}^{3-} \quad$ [14] and hence provides a protective property against demineralized lesions [15]. PRG Barrier Coat ${ }^{\circledR}$ improved remineralization of eroded enamel in the present study, supporting previous reports. Although enamel specimens were eroded in the present study, S-PRG fillers were reported to have additionally reduced biofilm formation [11] [16] [17].

Glass ionomer cements have been used in pediatric restorative dentistry for many years because of favorable properties such as biocompatibility, chemical adhesion to dentin and enamel, a coefficient of thermal expansion similar to that of tooth structure, versatility, and most importantly, its fluoride-releasing property [18]. FujiVII ${ }^{\circledR}$ is a newly developed type of glass ionomer cement whose flowability has been greatly improved, facilitating close contact between the tooth surface and the cement and thereby resulting in an improved remineralization effect by efficiently providing fluoride to enamel.

QLF-D is a device used to evaluate severity or the time-course change of the early demineralized lesion by calculating mineral density [6] [7]. When a tooth is lit in a blue light, fluorescence is reflected especially on the enamel-dentin junction. If a demineralized lesion exists in the enamel, the volume of reflected fluorescence is reduced. QLF-D senses the difference in reflected fluorescence in the volume between the unaffected and demineralized regions, detects dimensions of the region where the decreased fluorescence is observed, and then calculates mineral density in the demineralized lesion ( $\Delta \mathrm{Q}$ value) by multiplying the rate of decrease in fluorescence (expressed in a negative value) and the dimensions of the region where the decreased fluorescence was observed (pixels). Hence, the $\Delta \mathrm{Q}$ values in the current study were expressed as negative values accompanying the unit of "\%pixels." As the QLF-D facilitates a noninvasive method of evaluating enamel density, it is applied in both in vivo and in vitro studies all over the world [7] [19].

Bovine enamel is used in general studies on demineralization [20] and remineralization [21]. In addition to the general rules applicable to studies within this field, bovine enamel was used in the present study because the donor cows were fed in the same surroundings and were sacrificed at almost the same age. It was expected that the same feeding condition would lead to very similar properties among enamel specimens in terms of acid resistance. However, the result indicated that $\Delta \mathrm{Q}$ value varied markedly between evaluations on PRG Barrier Coat $^{\circledR}$ and FijiVII ${ }^{\circledR}$. Since the bovine teeth used in the evaluation on PRG Barrier Coat ${ }^{\circledR}$ and FijiVII ${ }^{\circledR}$ were purchased at discrete days from the same meat trader, the results suggested that acid resistance might vary more from cow to cow than has been reported in the past.

\section{Conclusions}

1) Both PRG Barrier Coat ${ }^{\circledR}$ and FijiVII ${ }^{\circledR}$ offered improved remineralization of initially demineralized enamel in comparison with their corresponding conventional products.

2) Acid resistance of bovine enamel varies from donor to donor even the donors were fed and sacrificed under very similar/same conditions.

\section{Acknowledgements}

This manuscript was subsidized by JSPS KAKENHI Grant Number 15K20607. 


\section{References}

[1] Watanabe, K., Tanaka, T., Maki, K., Nakashima, H. and Watanabe, S. (2015) Amount of Calcium Elution and Eroded Lesion Depth in Bovine Enamel Derived from Single Short Time Immersion in Carbonated Soft Drink in Vitro. Open Journal of Stomatology, 5, 80-86. http://dx.doi.org/10.4236/ojst.2015.53012

[2] Khamverdi, Z., Vahedi, M., Abdollahzadeh, S. and Ghambari, M.H. (2013) Effect of a Common Diet and Regular Beverage on Enamel Erosion in Various Temperatures: An In-Vitro Study. Journal of Dentistry of Tehran University of Medical Sciences, 10, 411-416.

[3] Rao, B.S., Moosani, G.K., Shanmugaraj, M., Kannapan, B., Shankar, B.S. and Ismail, P.M. (2015) Fluoride Release and Uptake of Five Dental Restoratives from Mouthwashes and Dentifrices. Journal of International Oral Health, 7, $1-5$.

[4] Hosoya, Y., Ando, S., Otani, H., Yukinari, T., Miyazaki, M. and Garcia-Godoy, F. (2013) Ability of Barrier Coat S-PRG Coating to Arrest Artificial Enamel Lesions in Primary Teeth. American Journal of Dentistry, 26, $286-290$.

[5] Ma, S., Imazato, S., Chen, J.H., Mayanagi, G., Takahashi, N., Ishimoto, T. and Nakano, T. (2012) Effects of a Coating Resin Containing S-PRG Filler to Prevent Demineralization of Root Surfaces. Dental Materials Journal, 31, 909-915. http://dx.doi.org/10.4012/dmj.2012-061

[6] Ko, H.Y., Kang, S.M., Kim, H.E., Kwon, H.K. and Kim, B.I. (2015) Validation of Quantitative Light-Induced Fluorescence-Digital (QLF-D) for the Detection of Approximal Caries in Vitro. Journal of Dentistry, 43, 568-575. http://dx.doi.org/10.1016/j.jdent.2015.02.010

[7] Kim, H.E., Kwon, H.K. and Kim, B.I. (2013) Recovery Percentage of Remineralization According to Severity of Early Caries. American Journal of Dentistry, 26, 132-6.

[8] Murayama, R., Furuichi, T., Yokokawa, M., Takahashi, F., Kawamoto, R., Takamizawa, T., Kurokawa, H. and Miyazaki, M. (2012) Ultrasonic Investigation of the Effect of S-PRGfiller-Containing Coating Material on Bovine Tooth Demineralization. Dental Materials Journal, 31, 954-959. http://dx.doi.org/10.4012/dmj.2012-153

[9] Lussi, A. and Carvalho, T.S. (2015) The Future of Fluorides and Other Protective Agents in Erosion Prevention. Caries Research, 49, 18-29. http://dx.doi.org/10.1159/000380886

[10] Mielczarek, A., Gedrange, T. and Michalik, J. (2015) An in Vitro Evaluation of the Effect of Fluoride Products on White Spot Lesion Remineralization. American Journal of Dentistry, 28, 51-56.

[11] Creeth, J.E., Kelly, S.A., Martinez-Mier, E.A., Hara, A., Bosma, M.L., Butler, A., Lynch, R.J. and Zero, D.T. (2015) Dose-Response Effect of Fluoride Dentifrice on Remineralisation and Further Demineralisation of Erosive Lesions: A Randomised in Situ Clinical Study. Journal of Dentistry, 43, 823-831.

[12] Lippert, F., Butler, A., Lynch, R.J. and Hara, A.T. (2012) Effect of Fluoride, Lesion Baseline Severity and Mineral Distribution on Lesion Progression. Caries Research, 46, 23-30. http://dx.doi.org/10.1159/000334787

[13] ten Cate, J.M., Exterkate, R.A. and Buijs, M.J. (2006) The Relative Efficacy of Fluoride Toothpastes Assessed with pH Cycling. Caries Research, 40, 136-141. http://dx.doi.org/10.1159/000091060

[14] Mungara, J., Philip, J., Joseph, E., Rajendran, S., Elangovan, A. and Selvaraju, G. (2013) Comparative Evaluation of Fluoride Release and Recharge of Pre-Reacted Glass Ionomer Composite and Nano-Ionomeric Glass Ionomer with Daily Fluoride Exposure: An in Vitro Study. Journal of Indian Society of Pedodontics and Preventive Dentistry, 31, 234-239. http://dx.doi.org/10.4103/0970-4388.121820

[15] Iijima, M., Ito, S., Nakagaki, S., Kohda, N., Muguruma, T., Saito, T. and Mizoguchi, I. (2014) Effects of Immersion in Solution of an Experimental Toothpaste Containing S-PRG Filler on Like-Remineralizing Ability of Etched Enamel. Dental Materials Journal, 33, 430-436. http://dx.doi.org/10.4012/dmj.2013-224

[16] Yoneda, M., Suzuki, N., Masuo, Y., Fujimoto, A., Iha, K., Yamada, K., Iwamoto, T. and Hirofuji, T. (2012) Effect of S-PRG Eluate on Biofilm Formation and Enzyme Activity of Oral Bacteria. International Journal of Dentistry, 2012, Article ID: 814913. http://dx.doi.org/10.1155/2012/814913

[17] Hahnel, S., Wastl, D.S., Schneider-Feyrer, S., Giessibl, F.J., Brambilla, E., Cazzaniga, G. and Ionescu, A. (2014) Streptococcus mutans Biofilm Formation and Release of Fluoride from Experimental Resin-Based Composites Depending on Surface Treatment and S-PRG Filler Particle Fraction. The Journal of Adhesive Dentistry, 16, 313-321.

[18] Berg, J.H. and Croll, T.P. (2015) Glass Ionomer Restorative Cement Systems: An Update. Pediatric Dentistry, 37, 116-124.

[19] Jo, S.Y., Chong, H.J., Lee, E.H., Chang, N.Y., Chae, J.M., Cho, J.H., Kim, S.C. and Kang, K.H. (2014) Effects of Various Toothpastes on Remineralization of White Spot Lesions. The Korean Journal of Orthodontics, 44, 113-118. http://dx.doi.org/10.4041/kjod.2014.44.3.113

[20] Nassur, C., Pomarico, L. and Maia, L.C. (2013) Reliability Analysis of two Methods for Measuring Active Enameldemineralization: An in Vitro Study. European Journal of Dentistry, 7, 159-164. http://dx.doi.org/10.4103/1305-7456.110157 
[21] Gmür, R., Giertsen, E., van der Veen, M.H., de Josselin de Jong, E., ten Cate, J.M. and Guggenheim, B. (2006) In Vitro Quantitative Light-Induced Fluorescence to Measure Changes in Enamel Mineralization. Clinical Oral Investigations, 10, 187-195. http://dx.doi.org/10.1007/s00784-006-0058-z 